\title{
MINIMUM TORQUE CHANGE MODEL VS. POWER LAW FOR ELLIPTIC MOVEMENTS
}

\section{TAKASHI OYAMA}

Faculty of Computer Science and System Engineering, Okayama Prefectural University, Japan

ABSTRACT
This study compared between the minimum torque change model and the power law model, for human elliptic
movements. In simulations, trajectories based on the two models were computed, at different movement velocities and
joint viscosities. The computed trajectories, based on the power law model could reproduce the characteristics of actual
human movements, better than the other model. The results suggest the criteria of computational simplicity, in addition
to the optimization of smoothness in human motor planning.
KEYWORDS: Motor Control, Minimum Torque Change Model \& Power Law

Received: Jun 01, 2017; Accepted: Jun 20, 2017; Published: Oct 03, 2017; Paper Id.: IJBTROCT20172

\section{INTRODUCTION}

\section{Background}

A number of models have been proposed, to explain the human motor control system. It is thought that, human movements exhibit robust similarity over trials, because the human motor control system plans and produces the trajectory of a movement, according to some universal principles and criteria.

Movement smoothness has been proposed, as one of the criteria respected by the human motor control system. A minimum jerk model has been proposed that quantifies smoothness, as the function of the jerk (the third derivative of position), which can reproduce the characteristics of human movements (Flash \& Hogan, 1985). A minimum torque change (MTC) model has been also proposed under the assumption that, humans produce smooth movements of which derivative of the joint torques are optimized, as the quantification of smoothness (Uno et al., 1989). The MTC model takes into account, not only the kinematics, but also the kinetic information on human biomechanics; therefore, it can reproduce the characteristics of human movements better than the minimum jerk model, for many movement situations.

On the other hand, for elliptic movements, the power law relation between the velocity and curvature of a trajectory has been demonstrated (Lacquaniti et al., 1983; de'Sperati\& Viviani, 1997; Catavitello et al., 2016). The model based on the power law relation, does not explicitly take into account the smoothness of movements. Some studies have reported that, trajectories based on the minimum jerk model reproduce the power law relation, moreover the power law relation is the result of the criterion of movement smoothness (Wann et al., 1998; Richardson \& Flash, 2002). However, it has not been determined whether the criterion of smoothness is rigidly equivalent, to the power law.

In this study, measurement and computer simulation experiments were examined. Measured trajectories of 
movements, by performing human subjects and computed trajectories based on the MTC and power law models were compared to elliptic movements, to determine the appropriate criteria used by the human motor control system.

\section{METHODS}

\subsection{Minimum Torque Change Model}

The minimum torque change (MTC) model quantifies the smoothness of movements, as the function of the derivative of the joint torques. The model serves a trajectory of which the value of the criteria function is minimized, under the given boundary conditions. The criteria function is expressed as Equation (1):

$$
\frac{1}{2} \int_{0}^{t_{f}} \sum_{i} \dot{\tau}_{i} d t
$$

Where the beginning and the end of a movement are assumed to be at $t=0$ and $t_{f}$, respectively. $\tau_{i}$ denotes the torque exerted by the $i$-th joint, relating to the movement.

For a reaching movement between the beginning and end points, a trajectory based on the MTC model can be computed, by giving the two points as the boundary conditions. For a curved trajectory like an elliptical movement, some via-points are aligned to compute a curved trajectory based on the MTC model. In this study, three via-points were aligned at the edges of the ellipse, where the value of the trajectory curvature was extremal.

\subsection{Power Law Model}

For repetitive elliptic movements, the relation between velocity $v(t)$ and curvature $c(t)$ of a movement trajectory are approximately expressed as Equation (2):

$$
\log v \cong \alpha \log |c|+\beta
$$

Where $\alpha$ and $\beta$, denote linear regression coefficients. $\alpha$ is a negative number of natural human movements: the deceleration at tight curves and acceleration, at gradual curves for human movements. The value of $\alpha$ is between-0.4 to -0.25 . In particular, $\alpha$ is nearly $-\frac{1}{3}$, for fast and smooth repetitive elliptic movements, produced by human subjects.

In Cartesian coordinates, the velocity $v(t)$ and curvature $c(t)$ of a trajectory are expressed as Equation (3), according to their definitions:

$$
c=\frac{\dot{x} \ddot{y}-\ddot{x} \dot{y}}{v^{3}}
$$

When a path of a trajectory is expressed by using an intervening variables $(t)$, and an elliptic curve is specified as the path of the trajectory, where $x(t)=\mathrm{a} \cos (s), y(t)=\mathrm{b} \sin (s)$, the relationship between velocity and curvature is expressed as Equation (4), by modifying Equation (3):

$$
c=\frac{\mathrm{ab} \cdot \dot{s}^{3}}{v^{3}}
$$

When $\dot{s}$ is constant at the trajectory, Equation (4) is equivalent to Equation (2), where the value of the parameter $\alpha$ rigidly equals to $-1 / 3$. For elliptic movements, the power law model setting $\alpha=-1 / 3$, means the constancy of $s$ along an elliptic curve. It can be confirmed by using a variational method that, the trajectory with the parameter $\alpha$ is set to-1/3, is very similar to the computed trajectory, based on the model to optimize the criteria function of movement smoothness (e.g. minimum jerk model), although they are not identical except when a equals to $b$, in the equation of an elliptic curve. The 
difference between the power law model and the smoothness criteria optimization model, suggests an appropriate criterion other than smoothness for the human motor control system.

\subsection{Measurement Experiment}

Six healthy right-handed persons participated in this experiment. The subjects were asked to trace an elliptic curve printed on a paper on a desk with their right hand. They showed ellipse was a horizontal ellipse: the length of its major axis was $15 \mathrm{~cm}$, the length of its minor axis was $4 \mathrm{~cm}$ and its center was aligned at the medial line of the subjects. The subjects had a wooden stick, attached to a position sensor (LIBERTY, Polhemus). The subjects' hand positions, during movements were measured at $144 \mathrm{~Hz}$.

Three levels of velocities were imposed on the subjects. They were instructed as follows: "move as precisely as possible" (slow condition), "move like in daily life" (medium condition), and "move as fast as possible" (fast condition). The subjects performed 30 periods of repetitive elliptic movements for each condition.

\subsection{Signal-Dependent Noise}

Harris \& Wolpert (1998) reported that, the human motor control system produces a movement such that, the variability of movement endpoints decreases under the assumption of signal-dependent noise (SDN), adding to motor commands during movement execution, whose amplitude increases with the amplitude of a source signal. Researcher van Beers et al. (2004) reported that, the variability of reaching movements between two points, performed with human subjects can be reproduced by hypothesizing SDN; trajectories produced with large torques involve large variability at the movement endpoint.

The $\operatorname{SDN} \sigma_{\mathrm{SDN}}$ adding to motor commands $u(t)$ is defined by Equation (5):

$$
\sigma_{\mathrm{SDN}}=N\left(0, k_{\mathrm{SDN}}^{2} \cdot u^{2}\right)
$$

Where, $N\left(\mu, \sigma^{2}\right)$ denotes a Gaussian distribution, and $k_{\mathrm{SDN}}$ denotes the parameter to modulate the magnitude of the SDN. The motor commands $u(t)$ are defined by Equation (6):

$$
u(t)=t_{e} t_{a} \ddot{\tau}(t)+\left(t_{e}+t_{a}\right) \dot{\tau}(t)+\tau(t)
$$

Where, $t_{e}$ and $t_{a}$ denote time constants of 30 and $40 \mathrm{~ms}$, and $\tau(t)$ denotes the joint torque of a corresponding joint (van Beers, 2004).

\section{RESULTS}

In the analysis, the relationship between the velocity and curvature of the trajectories was assessed according to Equation (2). A parameter $\alpha$ for linear regression and a correlation coefficient, $r$, were calculated for each trajectory.

\subsection{Measurement Data}

The $\alpha, r$, and mean velocity of the measured trajectories in three conditions of velocities (slow, medium, and fast) are illustrated in Figure 1.

The ANOVA on the $\alpha, r$, and mean velocity involving the Velocity Condition (slow, medium, and fast), as a factor revealed the significant main effects of the Velocity Condition on $\alpha, r$, and mean velocity (all $p<0.001$ ). Tukey's multiple comparison test revealed no significant differences, between slow and medium conditions on $\alpha(p=0.263)$, and 
significant differences in all other combinations on $\alpha, r$, and mean velocity (all $p<0.001$ ).
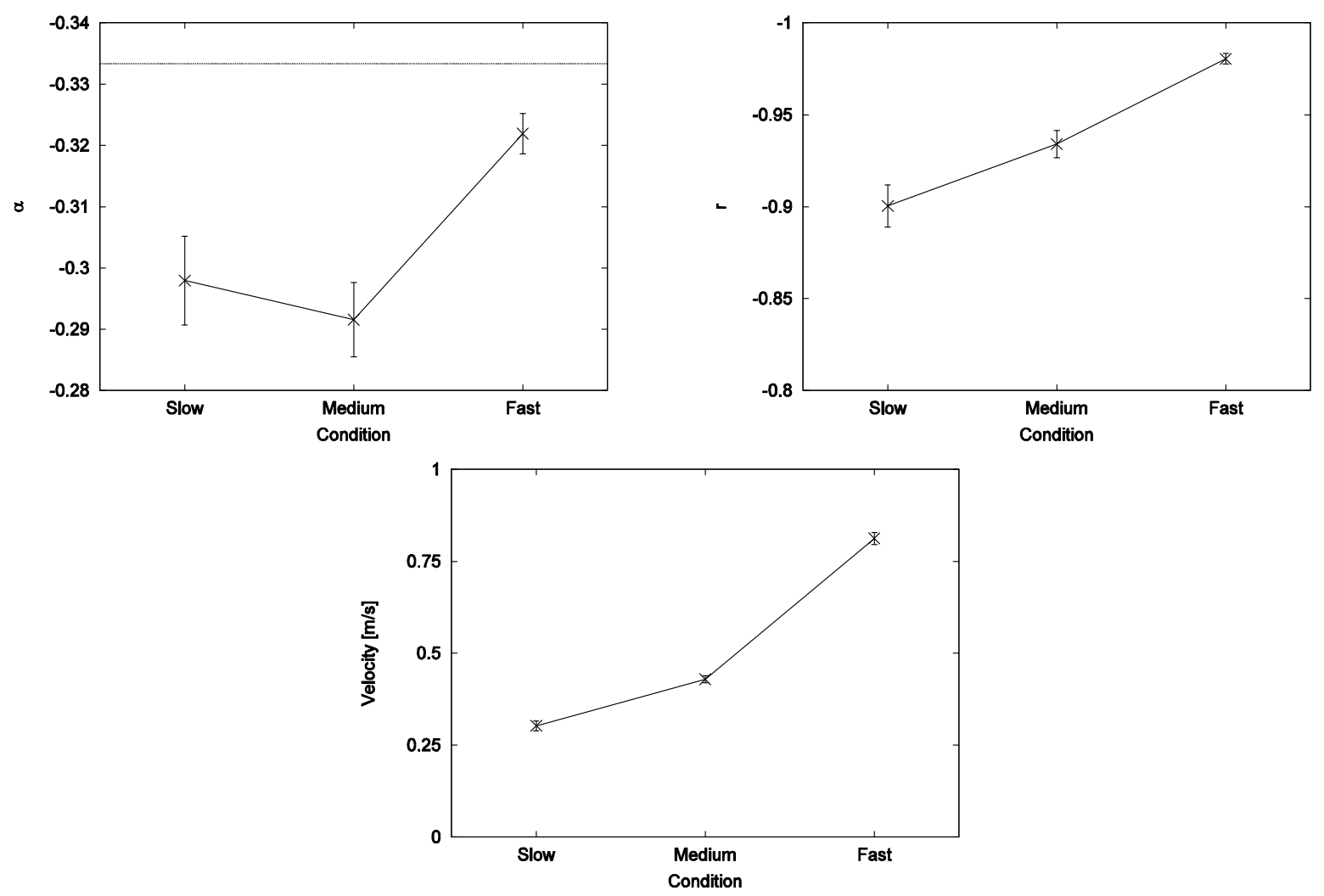

Figure 1: $\alpha, r$, and Velocity of Measured Trajectories in Three Conditions of Velocities.

The Dotted Line in the Figure for $\alpha$ Indicates-1/3.

Error Bars Indicate Standard Errors (the Same Hereinafter)

The results of the ANOVA, for the trajectory data of actual human movements suggest that, the value of parameter $\alpha$ approaches $-1 / 3$ in the fast condition, and the correlation coefficient, $|r|$, approaches to 1 in the fast condition, namely, the fit of the power law is improved by the increase in movement velocity.

\subsection{Trajectories Based on the MTC Model}

Trajectories of elliptic movements, based on the MTC model were computed at different velocities to investigate the capability of the MTC model, to imitate actual human movements in the parameters $\alpha$ and $r$ of the power law relation.

In this simulation, the dynamic parameters of a human arm (mass, inertia, length, and center of mass of an upper arm and a forearm) were defined, according to van Beers et al. (2004). The joint viscosity was examined at three values: $0.4,0.8$, and $1.2 \mathrm{Nm} /(\mathrm{rad} / \mathrm{s})$. Three movement durations (slow of $1200 \mathrm{~ms}$, medium of $760 \mathrm{~ms}$, and fast of $500 \mathrm{~ms}$ ) were examined that, corresponded to the velocity condition of the measurement experiment. The values of the movement durations were defined from the means of the movement durations of the measured trajectories, for each condition.

The $\alpha$ and $r$ of the trajectories, based on the MTC model for each condition of the joint viscosities and movement durations are illustrated in Figure 2. Note that, the MTC model gives the unique trajectory, for the same condition of a movement, since there were no error bars in the figures.

For all conditions, the parameter $\alpha$ was close to $-1 / 3$, and the correlation coefficient, $|r|$, was close to 1 . The interest of this study, is the imitation capability of the movement smoothness optimization model. For the computed 
trajectories, with the adequate joint viscosity value of 0.8 (van Beers, 2004), the Model (actual and MTC) $\times$ Velocity (slow, medium, and fast) ANOVA on the $\alpha$ and $r$, revealed significant interaction effects of Model $\times$ Velocity for both $\alpha$ and $r$ (both $p<0.001$ ). Thus, the MTC model could not imitate the influence of movement velocity on the power law relation between velocity and curvature, shown in actual human movement data.

\subsection{Influence of SDN on Trajectories based on the MTC and Power Law Models}

In Section 3.1, the decrease of the correlation coefficient, $|r|$ of the power law for slow movements performed with human subjects was shown. In this experiment, the computer simulation using SDN was examined to determine the reason for the phenomenon.
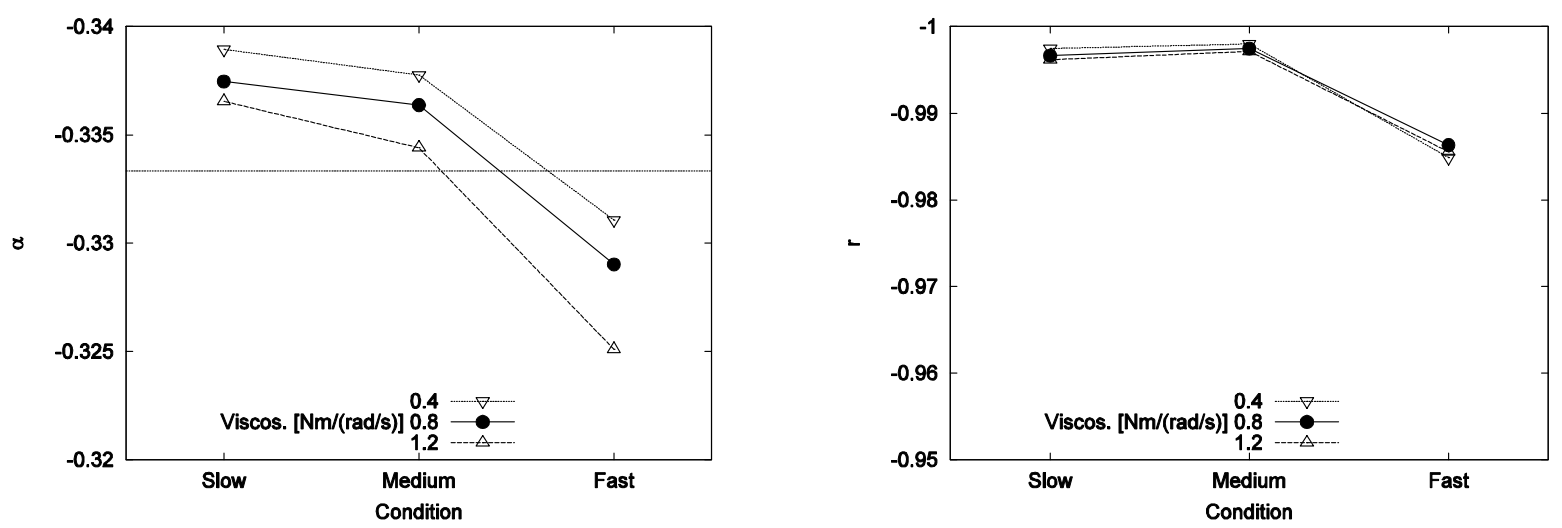

Figure 2: $\alpha$ and $r$ of Computed Trajectories Based on the MTC Model

The computer simulation using SDN was executed in almost the same manner as that, proposed by van Beers et al. (2004). Base trajectories deformed by adding SDN were computed, based on the MTC and power law models. A trajectory based on the power law model was computed, by setting the derivative of the intervening variable $s(t)$ constant, during a movement.

The magnitude of the noise $k_{\mathrm{SDN}}$ was set to $0.1,0.3$ and 0.5 . Multiple joint viscosities $0.2,0.4, \ldots, 1.2 \mathrm{Nm} /(\mathrm{rad} / \mathrm{s})$ were examined, because it is difficult to estimate the correct value of the joint viscosity and it can be modified, during the execution of a movement to effectively accomplish a task (Gomi, 1998). Two hundred trajectories were computed by adding SDN to the trajectories, based on the MTC and power law models for each condition, and the parameter $\alpha$ and $r$ of the trajectories deformed by adding SDN were analyzed.

Only the results for $k_{\mathrm{SDN}}$ set to 0.5 are illustrated in this paper, because of space limitations. The $\alpha$ and $r$ of trajectories, based on the power law model deformed by adding SDN are illustrated in Figure 3. The Magnitude of Noise $(0.1,0.3$, and 0.5$) \times$ Viscosity $(0.2,0.4, \ldots$, and 1.2) $\times$ Velocity (slow, medium, and fast) ANOVA, on $\alpha$ and $r$ revealed the significant main effects of Magnitude of Noise, Viscosity, and Velocity, and the significant interaction effects of Magnitude of Noise $\times$ Viscosity, Magnitude of Noise $\times$ Velocity, Viscosity $\times$ Velocity, and Magnitude of Noise $\times$ Velocity $\times$ Viscosity (all $p<0.001)$ 

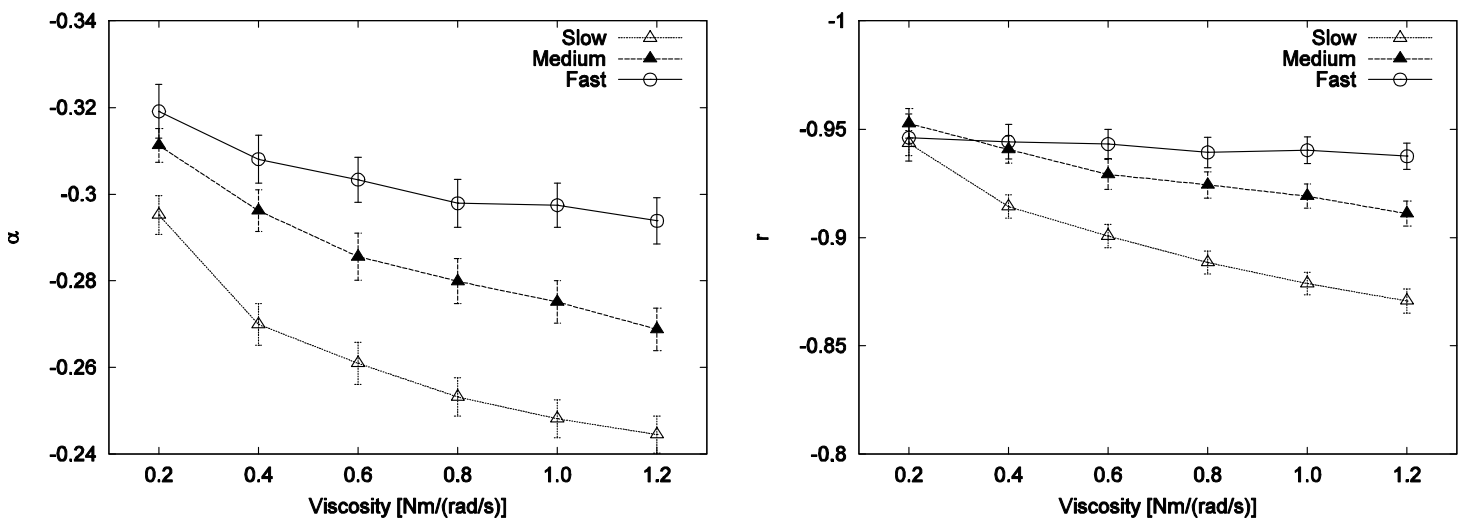

Figure 3: $\alpha$ and $r$ of Computed Trajectories Based on the Power Law Model Deformed by Adding SDN $\left(k_{\mathrm{SDN}}=0.5\right)$

The $\alpha$ and $r$ of trajectories, based on the MTC model deformed by adding SDN are illustrated in Figure 4. The Magnitude of Noise $(0.1,0.3$, and 0.5$) \times$ Viscosity $(0.2,0.4, \ldots$, and 1.2$) \times$ Velocity (slow, medium, and fast) ANOVA on $\alpha$ and $r$ was revealed significant main effects of Magnitude of Noise, Viscosity, and Velocity, and significant interaction effects Magnitude of Noise $\times$ Viscosity, Magnitude of Noise $\times$ Velocity, Viscosity $\times$ Velocity, and Magnitude of Noise $\times$ Velocity $\times$ Viscosity (all $p<0.001)$.
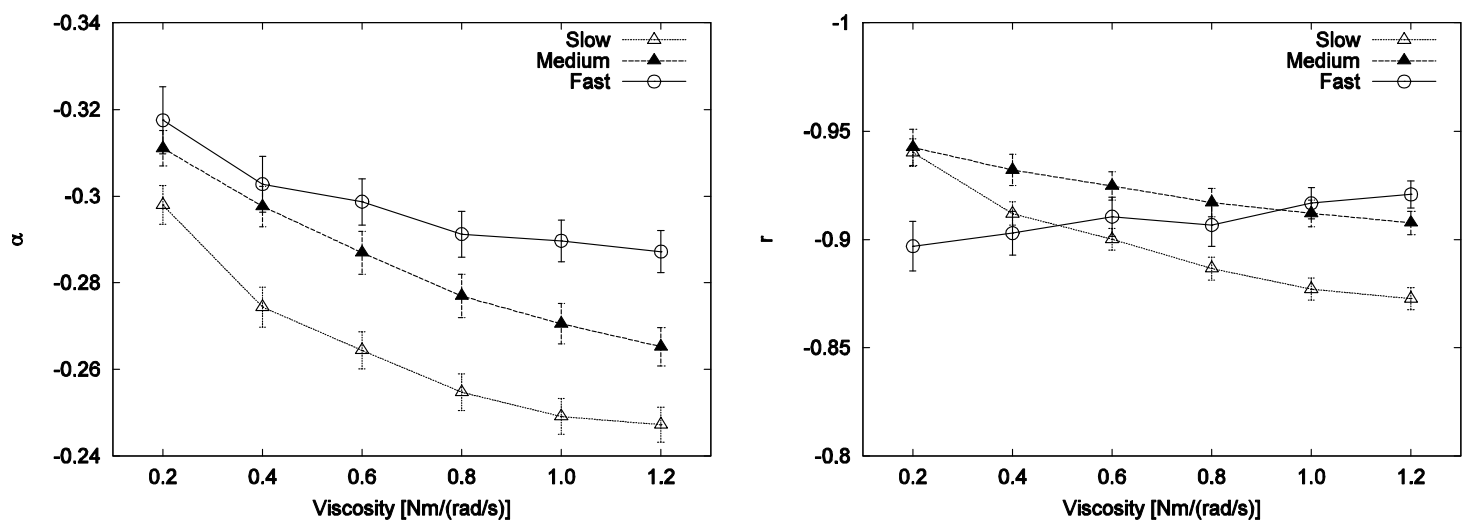

Figure 4: $\alpha$ and $r$ of Computed Trajectories Based on the MTC Model Deformed by Adding SDN $\left(k_{\text {SDN }}=0.5\right)$

The Model $($ MTC and power law) $\times$ Magnitude of Noise $(0.1,0.3$, and 0.5$) \times$ Viscosity $(0.2,0.4, \ldots$, and 1.2$) \times$ Velocity (slow, medium, and fast) ANOVA on $\alpha$ and $r$, where some results of interest in this study were shown, revealed the significant interaction effects of Model $\times$ Viscosity $\times$ Velocity (both $p<0.001$ ), and the insignificant interaction effects of Model $\times$ Magnitude of Noise $\times$ Viscosity $(p=0.183,0.151)$ and Model $\times$ Magnitude of Noise $\times$ Viscosity $\times$ Velocity ( $p=0.721,0.269$ ). Tukey's HSD multiple comparison on $r$, revealed significant differences between medium and fast Velocity, for the MTC model at $0.2,0.4,0.6,0.8$, and 1.0 Viscosity (all $p<0.001$ ), and an insignificant difference between medium and fast Velocity, for the MTC model at 1.2 Viscosity $(p=0.277)$.

The factors of Magnitude of Noise and Viscosity were neglected, to simplify the comparison between the two models. The $\alpha$ and $r$ of trajectories, based on the two models added SDN are illustrated in Figure 5, where $k_{\text {SDN }}$ and the joint viscosity were set to 0.5 and 0.8 , respectively. The Model (MTC and power law) $\times$ Velocity (slow, medium, and fast) 
ANOVA on $\alpha$ revealed a significant main effect of Velocity $(p<0.001)$, an insignificant main effect of Model $(p=$ $0.184)$, and an insignificant interaction effect of Model $\times$ Velocity $(p=0.253)$. The Model (MTC and power law) $\times$ Velocity (slow, medium, and fast) ANOVA, on $r$ revealed significant main effects of Model and Velocity, and a significant interaction effect of Model $\times$ Velocity (all $p<0.001)$. Tukey's multiple comparison test revealed in significant differences between the two models in slow $(p=0.999)$ and medium $(p=0.660)$ conditions, and a significant difference between the two models in the fast condition $(p<0.001)$. The $|r|$ consistently approached 1 with the movement velocity for the power law model; conversely, it was not so for the MTC model.
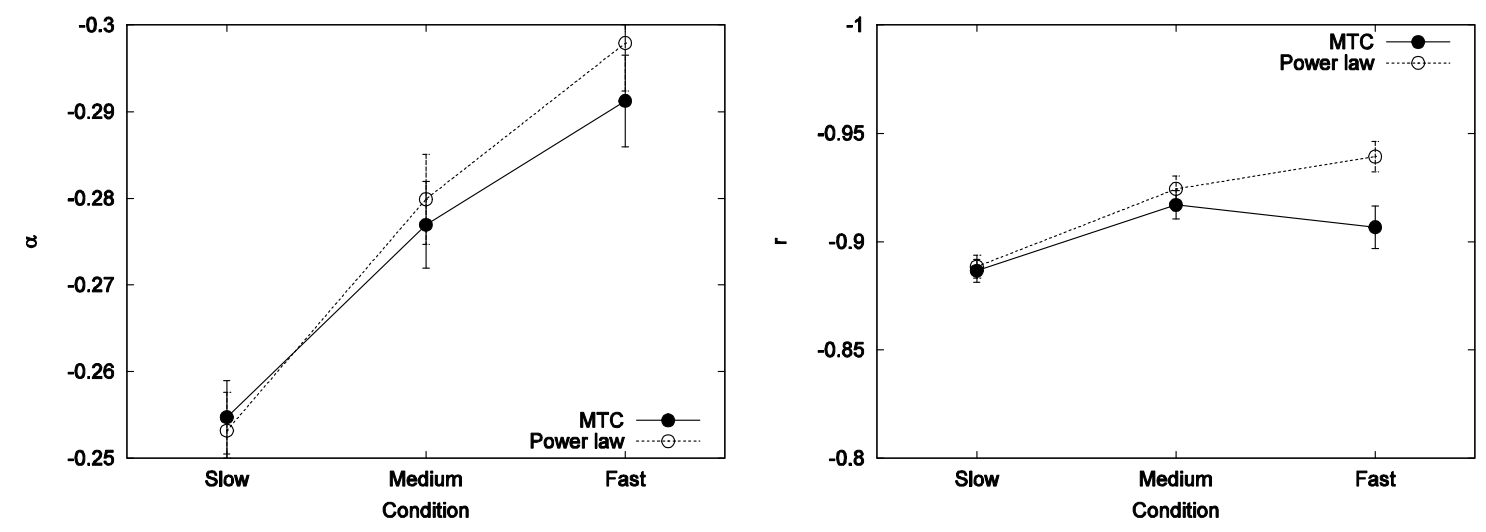

Figure 5: $\alpha$ and $r$ of Computed Trajectories Based on the MTC and Power Law Model Where the Parameters $\boldsymbol{k}_{\mathrm{SDN}}$ and Joint Viscosity were Set 0.5 and 0.8

\section{DISCUSSIONS}

The purpose of this study is to compare the two models of human motor control system. One was based on the model optimizing the criteria function of movement smoothness, and the other with respect to the kinematic relation between velocity and curvature of a movement trajectory. The two models were compared in the imitation capability of actual human movement characteristics. The parameters of the power law, that expresses the relation between velocity and curvature of a movement trajectory were assessed, to simplify the analysis.

The correlation coefficient, $|r|$ of the power law approached 1 with the movement velocity for measuring actual movements. In contrast, for the computed pure trajectories based on the MTC model, the $|r|$ decreased with the movement velocity, when the value of the joint viscosity set the adequate value, proposed in previous research (Nakano et al., 1999; van Beers et al., 2004). In the simulations using SDN, to determine the reasons for the characteristics of actual movements, computed trajectories, based on the MTC and power law models were deformed by adding SDN. For both models, the $|r|$ varied with the magnitude of noise, joint viscosities and movement velocities. When the value of the joint viscosity was set adequately, the power law model could reproduce the characteristics of actual movements, such that $|r|$ Consistently increased by movement velocity. On the other hand, for the MTC model, the influence of movement velocity on $|r|$ became like actual movements, only when too high joint viscosity was set. The results was that, the fit of the power law relation was better for the deformed trajectories based on the power law model, than for the MTC model may be sound obvious; however, what was emphasized by this study is the relationship between $|r|$ And the movement velocity, shown in the measurements and simulation, examining the power law model experiments. The simulations using SDN involved arm dynamics, since the results were not just derived from kinematic relation and were also not trivial.

The power law relation between the velocity and curvature of a trajectory, for elliptic movements means the 
constancy of the derivative of a time parameter, that expresses the path of a movement. If the human motor control system prefers the constancy of some parameters, for example thes Dealt with in this study, relevant to the production of a movement in motor planning to the optimization of criterion functions, that is often hard to achieve, it allows us to conjecture the easiness of computation (Karniel, 2013; Dounskaia, 2016), as the criterion that the human motor control system respects in motor planning. It has to be made clear, how such mathematical parameters that are assumed to be constant, during a movement are interpreted in the biological system.

\section{ACKNOWLEDGMENTS}

This work was supported by JSPS KAKENHI Grant Number 15K21301.

\section{REFERENCES}

1. Flash, T., \& Hogan, N. (1985).The coordination of arm movements: an experimentally confirmed mathematical model. Journal of Neuroscience, 5, 1688-1703.

2. Uno, Y., et al. (1989). Formation and control of optimal trajectory in human multi joint arm movement. Biological Cybernetics, 61, 89-101.

3. Lacquaniti, F., et al. (1983). The law relating the kinematic and figural aspects of drawing movements. ActaPsychologica, 54, $115-130$

4. de'Sperati, C., \& Viviani, P. (1997). The relationship between curvature and velocity in two-dimensional smooth pursuit eye movements. Journal of Neuroscience, 17, 3932-3945.

5. Catavitello, G., et al. (2016). Drawing ellipses in water: evidence for dynamic constraints in the relation between velocity and path curvature. Experimental Brain Research, 234, 1649-1657.

6. Wann J., et al. (1998). Relation between velocity and curvature in movement: equivalence and divergence between a power law and a minimum-jerk model, Journal of Experimental Psychology, 14, 622-637.

7. Richardson, M.J.E., \&Flash T. (2002). Comparing smooth arm movements with the two-thirds power law and the related segmented control hypothesis. Journal of Neuroscience, 22, 8201-8211.

8. Harris, C.M., \&Wolpert, D.M. (1998). Signal-dependent noise determines motor planning. Nature, 394, 780-784.

9. Van Beers, R.L., et al. (2004). The role of execution noise in movement variability. Journal of Neurophysiology, 91, $1050-1063$.

10. Gomi, H., \&Osu, R. (1998). Task-dependent viscoelasticity of human multijoint arm and its spatial characteristics for interaction with environments. Journal of Neuroscience, 18, 8965-8978.

11. Nakano, E., et al. (1999). Quantitative examinations of internal representations for arm trajectory planning: minimum commanded torque change model. Journal of Neurophysiology, 81, 2140-2155.

12. Karniel, A. (2013). The minimum transition hypothesis for intermittent hierarchical motor control. Frontiers in Computational Neuroscience, 7, doi: 10.3389/fncom.2013.0012.

13. Dounskaia, N., \&Shimansky, Y. (2016). Strategy of arm movement control is determined by minimization of neural effort for joint coordination. Experimental Brain Research, 234, 1335-1350. 